\title{
Sport et grossesse : une nécessaire prescription
}

\author{
Carole Maître
}

INSEP, 11, avenue du Tremblay, 75012 Paris, France

A paraître dans Science \& Sport, 2013

\section{Résumé}

La pratique sportive doit être encouragée au cours de la grossesse, car les bénéfices sur la santé maternelle et fœtale sont importants en particulier en prévention du diabète gestationnel et du surpoids. La pratique est d'intensité au moins modérée en fonction du niveau antérieur et peut être poursuivie sans risque au cours de la grossesse physiologique, en l'absence de contre-indications. La reprise dans le post-partum sera progressive et individualisée.

Mots clés : Grossesse ; Sport ; Activité physique ; Diabète gestationnel ; Gain de poids gestationnel ; Pré-éclampsie ; Post-partum

\section{Summary}

The sport should be encouraged during pregnancy because the health benefits are important, maternal and fetal especially in preventing gestational diabetes and overweight. Practice is at least moderate intensity depending on the previous level and can be continued safely during physiological pregnancy, in the absence of contraindications. Exercise in the postpartum period should increase gradually and should be individualized.

Keywords: Pregnancy ; Sport ; Physical activity ; Gestational diabetes ; Gestational weight gain ; Preeclampsia; Postpartum 
La pratique du sport chez la femme a augmenté ces dernières années, mais les femmes ne représentent encore que $37 \%$ environ des licenciés ; parmi les sports les plus représentés dans la population féminine licenciée figurent la danse, la gymnastique et l'équitation, mais il existe une pratique non encadrée hors fédération, avec des pratiques aussi diverses que la marche nordique, le yoga, la course à pied, aussi la question de la pratique du sport ou d'une activité physique se pose-t-elle naturellement avec une plus grande fréquence, pendant la grossesse, dans le cadre du suivi médical. Des recommandations tant de la Haute Autorité de santé que des Sociétés canadienne et américaine de gynécologie obstétrique permettent de faire avec chacune des patientes en fonction du type et du niveau de pratique un choix éclairé d'activités physiques ou sportives. Limiter la sédentarité pendant la grossesse et soutenir la reprise d'une activité dans la période du post-partum est essentiel pour éviter le surpoids, voire l'obésité qui est un problème majeur de santé publique.

\section{Un suivi des recommandations insuffisant}

En 2005, La Haute Autorité de santé confirme que commencer ou continuer une activité physique est possible, en dehors des activités à risque de chute ou de traumatisme et de la plongée sous-marine [1].

Ces recommandations qui se réfèrent à des études de cohortes multicentriques et des essais comparatifs se rapprochent des recommandations de la Société canadienne de gynécologie obstétrique (SCGO) et de l'American College of Obstetricians and Gynecologysts (ACOG) qui recommandent une activité physique de 30 minutes, au moins cinq fois par semaine, ou 150 minutes par semaine, d'intensité modérée, toujours en aérobie [2] and [3]. Ces préconisations concernent toutes les femmes y compris les femmes sédentaires avant leur grossesse, en l'absence de tout objectif de compétition.

L'activité physique ou sportive n'est pas à risque au cours de la grossesse physiologique, en l'absence de contre-indications obstétricales ou médicales absolues, elle est associée à une diminution des complications en particulier du diabète gestationnel et à un meilleur état de santé de la mère et de l'enfant. Mais, actuellement ces recommandations sont insuffisamment suivies.

Une étude danoise prospective, concernant 4718 femmes nullipares en cours de grossesse, montre une diminution significative en intensité et en durée de la pratique sportive, au cours des trois trimestres de la grossesse. Il y a une augmentation de la sédentarité passant de $6 \%$ à $29 \%$ dans cette population de femmes nullipares ; seules les femmes pratiquant une activité légère tendent à conserver leur niveau d'activité, cependant il est remarquable de noter qu'en réponse au programme danois d'information, incitatif sur l'activité physique ou sportive pendant la grossesse, un quart des femmes sédentaires avant la grossesse débute des activités dites légères, cette période étant initiatrice de modifications bénéfiques du comportement avec une meilleure perception des risques personnels, une attente de résultats positifs sur l'évolution de la grossesse, une responsabilisation vis-à-vis de l'enfant à naître, tous ces éléments contribuent à faciliter l'adhésion à des plans de prévention structurés [4].

L'étude Behavior Affecting Baby and You (BABY) concerne 110 femmes d'origine espagnole, d'âge jeune, incluses à la fin du premier trimestre, en surpoids ou obèses à risque de diabète gestationnel, randomisées en deux groupes, soit 58 femmes avec un programme d'exercices physiques associé à des conseils généraux d'éducation à la santé versus 52 femmes recevant des conseils généraux sans programme d'activité physique. Le suivi pendant la grossesse a montré une augmentation significative jusqu'au troisième trimestre de la pratique d'activité physique dans le groupe ayant un programme d'activités structurées avec un taux de satisfaction de $95 \%$, l'autre groupe diminuant la pratique d'activité physique [5]. 
D'autres facteurs interviennent sur la pratique en cours de grossesse : elle est d'autant plus faible que le niveau d'activité est faible à l'adolescence et l'année précédant la grossesse, l'âge jeune inférieur à 35 ans, l'indice de masse corporelle (IMC) supérieur à $25 \mathrm{~kg} / \mathrm{m} 2$, qu'il y a eu un traitement pour infertilité, un niveau d'études faible, des facteurs socioéconomiques peu élevés, un tabagisme actif, une alimentation riche en hydrates de carbones et comme l'a montré l'étude BABY une absence de programme d'activité physique adaptée à la grossesse [6] and [7].

\section{Des contre-indications peu nombreuses}

La grossesse pathologique et la grossesse à risque sont les seules contre-indications. Il peut d'agir de contre-indications absolues ou relatives (Tableau 1) qui impose dans ce dernier cas une évaluation de la balance bénéfice-risque médical ou obstétrical par l'équipe de suivi [2].

\section{Une physiologie favorable à la pratique sportive}

Le bilan des adaptations cardiovasculaires et respiratoires lors de la grossesse est favorable à la pratique sportive : l'augmentation du volume plasmatique atteint 40 à $50 \%$ du fait de l'unité fœtoplacentaire et de la stimulation du système rénine - angiotensine - aldostérone, le volume d'éjection systolique (VES) et la fréquence cardiaque (FC) augmentent respectivement de 30 à $50 \%$ jusqu'à 20 semaines de grossesse puis se stabilisent avec pour conséquence un débit cardiaque augmenté (de l'ordre de $40 \%$ ). Certaines modifications comme l'augmentation du VES perdure jusqu'à un an en post-partum. De plus, le volume érythrocytaire est augmenté de $20 \%$ en moyenne.

Il peut être constaté une consommation maximale d'oxygène augmentée de 10 à $30 \%$ environ, soutenue par une adaptation des capacités respiratoires avec une augmentation du volume courant liée à une stimulation des centres respiratoires par la progestérone avec une respiration plus ample sans augmentation de la fréquence respiratoire d'où une meilleure ventilation alvéolaire et une diminution de la $\mathrm{PaCo}$. Tous ces facteurs favorisent les activités en aérobie qui sont conseillées [8] and [9].

Ces bons atouts pour la pratique sportive sont contre-balancés dès le sixième mois par la prise de poids, le développement abdominal de l'utérus et la modification du centre de gravité, une lordose lombaire accentuée et une laxité ligamentaire augmentée d'origine hormonale (progestérone, relaxine) qui feront préférer dans le dernier trimestre les sports dits non portés.

\section{Des bénéfices nombreux}

La revue Cochrane publiée en 2006 retient 11 essais et concerne 472 femmes pratiquant une activité physique modérée au moins trois fois par semaine, elle montre une amélioration ou un maintien de la santé physique, avec une diminution du syndrome douloureux abdominal du quatrième mois et des lombalgies [10].

\subsection{Une prise de poids limitée}

Le surpoids au cours de grossesse est significativement moindre chez les femmes pratiquant une activité physique régulière les deux derniers trimestres par rapport aux sédentaires, le risque de poids excessif diminue avec le niveau d'activité physique $(\mathrm{p}<0,05)$ [11] ? Clapp notait déjà une prise de poids plus basse et un retour plus rapide au poids antérieur, si l'activité était poursuivie pendant le troisième trimestre [8]. L'activité physique ou sportive modérée et régulière pendant la grossesse est une prévention du risque ultérieur de surpoids voire d'obésité : chez une femme obèse, un gain de 11 à $15 \mathrm{~kg}$ multiplie par quatre le risque de majorer le poids initial à un an du post-partum $(+5 \mathrm{~kg})$ [12]. Mais l'activité seule ne suffit pas, l'étude Nutrition and Exercise Lifestyle Intervention Program 
(NELIP) a montré la réduction significative de la prise de poids maternelle par l'effet synergique d'un suivi nutritionnel ( $2000 \mathrm{kcal} /$ jour et $40-55 \%$ d'apports en hydrates de carbone) et d'un programme de marche au moins trois fois par semaine, avec une fréquence moindre des poids de naissance élevés (supérieur à 4-4,5 kg) par rapport aux femmes de même IMC n'ayant pas suivi le programme complet [13].

À côté des conséquences obstétricales avec une augmentation du nombre de césariennes et un risque accru de diabète gestationnel, une prise de poids excessive liée à la sédentarité a des conséquences néonatale et infantile sur le poids de naissance et l'obésité infantile, indépendamment des facteurs génétiques comme l'a montré l'analyse de cohorte de 513501 femmes et de leurs 1164750 nouveaunés avec une association constante et significative entre le gain pondéral pendant les grossesses et le poids de naissance des enfants dans une même fratrie [14].

\subsection{Sport et diabète gestationnel}

L'activité physique induit une amélioration de la sensibilité à l'insuline, une meilleure tolérance au glucose, retarde et diminue significativement le besoin en insuline. Qu'il s'agisse d'exercices en aérobie ou d'exercices contre-résistance, de renforcement musculaire des membres supérieurs qui sont plus faciles au troisième trimestre, l'activité physique ou sportive est un traitement adjuvant du diabète, elle sera associée à des recommandations nutritionnelles usuelles comme la fragmentation des apports en quatre repas minimum, l'exclusion des sucres d'absorption rapide, une portion d'hydrates de carbone à chaque repas; elle doit être précédée ou accompagnée d'une collation.

En prévention du diabète gestationnel, une pratique régulière au moins modérée a un effet bénéfique d'autant plus grand qu'elle fait suite à une pratique d'intensité vigoureuse l'année précédant la grossesse avec un OR $=0,49(0,24-1,01$ : IC95\%), ce bénéfice apparaît dès trois heures d'activités par semaine chez les femmes ayant un IMC normal ou en surpoids [15].

\subsection{Un bénéfice discuté sur la pré-éclampsie}

Actuellement, il n'est pas permis de conclure à un bénéfice significatif de l'activité physique débuté en cours de grossesse, mais il existe une diminution du risque chez les femmes pratiquant une activité physique régulière et vigoureuse l'année précédant la grossesse et poursuivie au début de la grossesse, ce qui s'explique par la complexité de l'étiopathogénie où intervient précocement, entre autre, la placentation et la réaction immunitaire [16] and [17].

Une augmentation légère du risque a été rapportée pour une activité physique du premier trimestre supérieure à quatre heures et demie par semaine ou plus de $40 \mathrm{MET} /$ heure par semaine ${ }^{1}$, ce qui correspond à plus d'une heure de vélo ou à 50 minutes de jogging par jour, avec comme hypothèse des modifications vasculaires et des interleukines modifiées par l'exercice physique [18]. La pratique d'une activité physique pour être bénéfique doit être encadrée par des conseils sur la fréquence, l'intensité et la durée et adaptée aux différents trimestres de la grossesse.

Note 1 Metabolite Equivalent Task (MET) équivaut à la dépense d'énergie du repos soit une consommation d'oxygène de $3,5 \mathrm{~mL} / \mathrm{kg}$ de masse corporelle et par heure.

\subsection{Une pathologie veineuse diminuée}

Les activités aquatiques diminuent les œdèmes des membres inférieurs, favorisant la redistribution de l'eau interstitielle vers le système vasculaire, améliore le retour veineux en diminuant la pression veineuse et le risque de survenue de varice. La prévention cardiovasculaire liée à l'activité physique 
est vrai aussi pendant la grossesse par voie directe et indirecte, en favorisant l'augmentation du HDL cholestérol et une diminution du surpoids [3].

\subsection{Un bien-être psychique}

L'activité physique diminue la fatigue et l'anxiété du premier trimestre et permet une meilleure adaptation aux modifications corporelles liées à la grossesse. Une diminution significative de la dépression du post-partum avec diminution des prescriptions médicamenteuses a été observée chez les femmes ayant une activité physique pendant la grossesse par rapport aux sédentaires OR $=0,81$ (IC95 $\%: 0,66-0,99)[19]$.

Certains bénéfices sont optimisés par une pratique d'activité sportive ou physique avant la grossesse et continuer pendant les trois trimestres de la grossesse, aussi est-il important d'apporter toute l'information utile en consultation dite préconceptionnelle ou au début de grossesse pour guider la femme dans son choix en évaluant son niveau d'activité (nombre d'heures, type, intensité) et les éventuelles contre-indications.

\section{Une obligation d'information en cours de grossesse}

L'information apportée a pour but une pratique sans risque et porte sur trois impératifs de la pratique [2] :

- l'apparition de tout symptôme impose l'arrêt de la pratique et un avis médical : métrorragies, dyspnée, contractions utérines, céphalées, vertiges, retard de croissance intra-utérin (RCIU), perte de liquide amniotique ;

- des règles de bonne pratique : hydratation complémentaire, apport énergétique adapté dès 13 semaines d'aménorrhée (SA), fonction de la dépense d'énergie, majorée en cas d'activité portée, une période d'échauffement progressif, un environnement aéré, pas d'exercice en décubitus dorsal à partir du quatrième mois de grossesse pour risque de compression de la veine cave par le volume utérin et pas d'exercices en altitude au-delà de $1800 \mathrm{~m}$;

- une fréquence, une intensité et une durée de l'activité physique ou sportive adaptée à la grossesse [2].

\subsection{Une dose d'activité physique adaptée}

\subsubsection{La fréquence des exercices}

La fréquence préconisée est de trois fois par semaine pour atteindre progressivement quatre à cinq fois par semaine, en fonction du niveau antérieur. Il est conseillé de ne pas faire d'activité physique vigoureuse deux jours de suite.

\subsubsection{Intensité des exercices}

L'intensité des exercices peut être appréciée par la FC, l'échelle de Borg ou le test de la parole qui paraît le plus simple en pratique. Les zones cibles sont modifiées par la grossesse, la réserve maximale de FC étant réduite.

Les recommandations sont de faire des exercices à 60 ou $70 \%$ de la $\mathrm{FC}$ maximale (FC maximale théorique : 220 ; âge), ce qui correspond à un niveau d'effort sans essoufflement important (Tableau 2). 
L'échelle de Borg peu utilisée en France, est une échelle de perception de l'effort ressenti pendant l'exercice, notée de 6 (0 effort) à 20 (effort maximal), la grossesse permet la zone 12-14 (effort modéré).

Le test de la parole détermine la zone d'intensité modérée : c'est le fait de mener une conversation normale sans essoufflement, pendant la pratique sportive.

\subsubsection{Durée des exercices}

La durée des exercices atteint progressivement 30 minutes par jour chez les femmes sédentaires, elle peut être de 30 à 40 minutes pour les sportives.

Chez la sportive de haut niveau, un programme d'entrainement associe renforcement musculaire et travail d'endurance en aérobie, avec une intensité qui peut être plus vigoureuse à $80 \%$ de la FC maximale en concertation entre le professionnel de santé, l'entraîneur, la sportive suivant ses objectifs et l'évolution normale de la grossesse [20]. Adapter les apports nutritionnels est utile pour éviter une prise de poids explosive en rapport avec une diminution franche de la dépense d'énergie ; les conditions d'une reprise à haut niveau seront alors réunies, favorisées par la bonne adaptation cardiovasculaire à l'effort persistant de six mois à un an dans la période du post-partum.

\subsection{Choix d'activité sportive}

Le choix d'activité sportive est large [8]. Parmi les activités recommandées :

- la marche reste l'activité la plus pratiquée ;

- la natation est un sport qui fait travailler les grands chaînes musculaires et peut être pratiqué jusqu'à terme ;

- le yoga adapté à la grossesse avec des exercices respiratoires et posturaux adaptés ;

- le vélo sur du plat est possible : un essai contrôlé à $70 \%$ de VO2max ne montre aucune différence dans le terme de la grossesse et le poids de naissance par rapport à des femmes sédentaires [8].

D'autres activités sont possibles en fonction du niveau de pratique antérieure : le jogging seulement jusqu'au cinquième mois sur terrain souple, l'équitation est discutée en fonction du niveau et en limitant les allures jusqu'au deuxième trimestre, de même les sports de montagne dont certains comme le ski alpin est possible au premier trimestre et d'autre comme le ski de fond peut être autorisé jusqu'au deuxième trimestre sur un faible dénivelé, le tennis dont la pratique en double est possible jusqu'au cinquième mois, le golf jusqu'au sixième au septième mois. Pour toutes ces activités s'appliquent les principes de fréquence, durée et intensité modérée.

\subsection{Une contre-indication}

La Société de physiologie et de médecine subaquatiques et hyperbares en langue française contreindique de façon temporaire mais absolue la plongée sous-marine car elle expose à un risque de fausse couche spontanée, de malformations foetales : cardiaque, aortique et des membres.

En cas de grossesse méconnue, avant les six SA, il n'y a pas de risque si les règles des paliers sont respectées ; entre six et $13 \mathrm{SA}$, il n'y a pas d'indication à une interruption médicale de grossesse, mais le suivi échographique est mis en place.

\section{Risques ou idées reçues}


Les risques ou idées reçues sont :

- un risque de fausse couche spontanée est rapporté pour une pratique de plus de sept heures par semaine, pratique intensive au cours du premier trimestre dans une étude danoise rétrospective, mais l'activité ne correspond pas à l'activité modérée des recommandations consensuelles et ce risque n'est pas observé après 16 semaines de grossesse [21] : une pratique modérée et régulière en respectant les contre-indications n'est pas cause de fausses couches spontanées ;

- un risque de RCIU et faible poids de naissance. Il n'existe aucune corrélation entre le niveau d'activité physique et un RCIU ou un poids de naissance faible c'est ce qui résulte de l'analyse de 79692 naissances danoises [22]. En revanche, un exercice régulier à raison de trois fois par semaine jusqu'à 17 et $30 \mathrm{SA}$ chez des nullipares réduit le risque de poids de naissance élevé respectivement de $23 \%$ à $28 \%$ [23];

- le risque de prématurité n'est pas augmenté, il est même rapporté une diminution du risque par rapport aux femmes sédentaires HR : 0,82 (IC95 \% : 0,76-0,88) ; différentes hypothèses sont proposées, diminution des interleukines pro inflammatoires et amélioration de la sensibilité à l'insuline [24];

- le risque de traumatisme abdominal lié au sport est très peu documenté. Chez les sportives, les blessures abdominales ou de la poitrine représentent moins de $2 \%$ des lésions. Les sports à risque de chute et de traumatisme, les sports de contact sont à éviter [25].

\section{L'accouchement et la durée du travail}

La pratique sportive raccourcit la durée du travail qui est d'autant plus courte que la VO2max est élevée à 35-37 SA [26]. Il n'y a pas plus d'extraction instrumentale ou de césarienne chez des sportives faisant des séances d'exercices de 35 minutes, trois fois par semaine entre 12 et 39 SA versus des prégnantes sédentaires.

\section{Le sport en période de post-partum}

Un délai de huit à dix semaines est à respecter avant une reprise progressive, facilitée par le maintien de l'augmentation du VES, du débit cardiaque et de l'amplitude respiratoire.

L'allaitement n'est pas modifié par l'activité physique ni en quantité ni en qualité, une baisse de la lactation peut être liée à une hydratation insuffisante et ou une alimentation mal adaptée à la dépense d'énergie. Précocement sont possibles des exercices statiques de rapprochement des grands droits, sans hyperpression abdominale, en décubitus, accompagnant un renforcement du périnée qui sera contrôlé avant toute reprise d'activité dynamique.

\section{Conclusion}

Que la femme soit sédentaire, déjà active ou sportive de haut niveau, la pratique sportive adaptée à l'état de grossesse doit être encouragée, pratique régulière, modulée en fonction du niveau antérieur à la grossesse, évoluant en fonction des trimestres de la grossesse, et choisie par la femme en concertation avec le professionnel de santé en respectant les règles de durée, fréquence et intensité modérée et l'objectif de plaisir, pour apporter tous les bénéfices attendus pendant cette période de la maternité.

\section{Déclaration d'intérêts}

L'auteur déclare ne pas avoir de conflits d'intérêts en relation avec cet article. 


\section{Références}

[1] Recommandations pour les professionnels de santé. Comment mieux informer les femmes enceintes. Document sur le site : www.has-sante.fr. Avril 2005.

[2] Directive clinique conjointe de la Société des gynécologues et obstétriciens du canada et de la Société canadienne de physiologie de l'exercice. JOGC 2003;129:1-66.

[3] R. Artal, M. O’Toole. Guidelines of the American College of Obstetricians and Gynecologists for exercise during pregnancy and the postpartum period. Br J Sports Med, 37 (2003), pp. 6-12

[4] H.K. Hagaard, P. Damm, M. Hedegaard et al. Sports and leisure time physical activity during pregnancy in nulliparous women. Matern Child Health J, 15 (6) (2011), pp. 806-813

[5] L. Chasan Taber, M. Silveira, B.H. Marcus, B. Braun, E. Stanek, G. Markenson. Feasibility and efficacity of a physical activity intervention among pregnant women: the Behaviors Affecting Baby and You (BABY) Study. J Phys Act Health, 8 (2) (2011), pp. 228-238

[6] L.A. Haakstad, N. Voldner, T. Herriksen, K. Bo. Why do pregnant women stop exercising in the third trimester? Acta Obstet Gynecol Scand, 88 (11) (2009), pp. 1267-1275

[7] S. Phelan. Pregnancy: a "teachable moment" for weight control and obesity prevention. Am J Obstet Gynecol, 202 (2) (2010), p. 135 [e1-e8]

[8] J.F. Clapp III. Exercise during pregnancy: a clinical update. Clin Sports Med, 19 (2) (2000), pp. 273-286

[9] K. Melzer, Y. Schutz, M. Boulvain, B. Kayser. Physical activity and pregnancy: cardiovascular adaptations, recommendations and pregnancy outcomes. Sports Med, 40 (6) (2010), pp. 493-507

[10] M.S. Kramer, S.W. Mac Donald. Aerobic exercise for women during pregnancy. Cochrane Data Base Syst Rev, 3 (2006), p. CD000180

[11] H. Jiang, X. Qian, M. Li et al. Can physical activity reduces excessive gestational weight gain? Findings from a Chinese urban pregnant women cohort study. Int J Behav Nutr Phys Act, 9 (2012), pp. 9-12

[12] K.K. Vesco, P.M. Dietz, J. Rizzo et al. Excessive gestational weight gain and postpartum weight retention among obese women. Obstet Gynecol, 114 (5) (2009), pp. 1069-1175

[13] M. Mottola, I. Giroux, R. Gratton et al. Nutrition and exercise prevent excess weight gain in overweight pregnant women. Med Sci Sports Exerc, 42 (2) (2010), pp. 265-272

[14] D.S. Ludwig, J. Currie. The association between pregnancy weight gain and birthweight: a within family comparison. Lancet, 376 (9745) (2010), pp. 984-990

[15] E. Oken, N. Yi. Associations of physical activity and inactivity before and during pregnancy with glucose tolerance. Obstet Gynecol, 108 (2006), pp. 1200-1207

[16] H.K. Hagaard, B. Otlesen, M. Hedegraard. The association between leisure time physical activity in the year before pregnancy and preeclampsia. J Obstet Gynaecol, 30 (1) (2010), pp. 21-24 
[17] S. Meher, L. Duley. Exercise or other physical activity for preventing preeclampsia and its complications. Cochrane Database Syst Rev, 19 (2) (2006), p. CD005942

[18] M.L. Osterdal, M. Stram. Does leisure time physical activity in early pregnancy protect against preeclampsia? Prospective cohort in Danish women. BJOG, 116 (1) (2009), pp. 98-107

[19] M. Strom, E. Mortensen, T.I. Halidorson et al. Leisure time physical activity in pregnancy and risk of postpartum depression: a prospective study in a large national birth cohort. J Clin Psychiatry, 70 (12) (2009), pp. 1707-1714

[20] K.R. Kardel. Effects of intense training during and after pregnancy in top level athletes. Scand J Med Sci Sports, 15 (2005), pp. 79-86

[21] M. Madsen, T. Jorgensen, M.L. Jensen. Leisure time physical exercise during pregnancy and the risk of miscarriage: a study within the Danish National Birth Cohort. BJOG, 114 (2007), pp. 14191426

[22] H.K. Hagaard, K. Petersson, M. Hedegaard et al. Sports and leisure time physical activity in pregnancy and birth weight: a population- based study. Scand J Med Sci Sports, 20 (1) (2010), pp. e96-e102

[23] K.M. Owe, W. Nystad, K. Bo. Association between regular exercise and excessive newborn birth weight. Obstet Gynecol, 114 (4) (2009), pp. 770-776

[24] M. Juhl, J. Obsen, P.K. Andersen et al. Physical exercise during pregnancy and the risk of preterm birth: a study within the Danish National Birth Cohort. Am J Epidemiol, 167 (7) (2008), pp. $859-866$

[25] C.F. Finch. The risk of abdominal injury to women during sport. J Sci Med Sport, 5 (1) (2002), pp. $46-54$

[26] K.R. Kardel, B. Johansen, N. Voldner. Association between aerobic fitness in late pregnancy and duration of labor in nulliparous women. Acta Obstet Gynecol Scand, 88 (8) (2009), pp. 948-952 
Tableau 1. Contre-indications à l'exercice physique pendant la grossesse.

\begin{tabular}{|c|c|}
\hline Contre-indications absolues & Contre-indications relatives \\
\hline Rupture des membranes & Grossesse gémellaire après la 28e SA \\
\hline Travail préterme & Antécédents de prématurité \\
\hline Perte de liquide amniotique & Fausses couches spontanées répétées \\
\hline Retard de croissance intra-utérin & Anémie sévère (hémoglobine $<10 \mathrm{~g} / \mathrm{L}$ ) \\
\hline Béance cervico-isthmique - cerclage & Malnutrition \\
\hline Placenta prævia après la 28e SA & $\begin{array}{l}\text { Troubles cardiovasculaires ou respiratoires légers } \\
\text { ou modérés }\end{array}$ \\
\hline \multicolumn{2}{|l|}{ Métrorragies } \\
\hline \multicolumn{2}{|l|}{ Hypertension gravidique et pré-éclampsie } \\
\hline \multicolumn{2}{|l|}{ Grossesse multiple $>3$ fotus } \\
\hline $\begin{array}{l}\text { Maladies cardiovasculaires et ou pulmonaires } \\
\text { graves }\end{array}$ & \\
\hline
\end{tabular}

D'après l'American College of Obstetricians Gynecologists.

SA : semaine d'aménorrhée.

Tableau 2. Zones cibles en fonction de l'âge maternel suivant les recommandations de la Société canadienne de physiologie de l'exercice [2].

\begin{tabular}{|l|l|}
\hline \multicolumn{2}{|c|}{ Fréquence cardiaque (btt/min) } \\
\hline Avant l'âge de 20 ans & $140-155$ \\
\hline De 20 à 29 ans & $135-150$ \\
\hline De 30 à 39 ans & $130-145$ \\
\hline 40 ans et plus & $125-140$ \\
\hline
\end{tabular}

